\title{
Protocolo de avaliação de habilidades pragmáticas de crianças com transtornos do espectro do autismo
}

\section{Assessment of pragmatic abilities of children with autism spectrum disorders}

Fernanda Dreux Miranda Fernandes ${ }^{1}$

\section{RESUMO}

Objetivo: Propor um protocolo de avaliação das habilidades pragmáticas da comunicação de crianças incluídas no espectro do autismo, aplicá-lo e comparar seus resultados com os do Perfil Funcional da Comunicação. Métodos: Participaram deste estudo 62 crianças entre 2 e 12 anos de idade, com diagnóstico incluído no espectro do autismo e sem perdas sensoriais ou síndromes genéticas diagnosticadas. A partir de amostras em vídeo, fonoaudiólogos responderam ao protocolo proposto e os resultados foram analisados em relação a protocolos já usados no serviço em que o estudo foi realizado. Resultados: Apenas os dados referentes à interatividade da comunicação e ao uso do meio comunicativo verbal apresentaram correlações significativas com o desempenho nos aspectos pragmáticos da linguagem, conforme verificado pelo protocolo proposto. O protocolo foi capaz de constatar que maiores possibilidades de interação com a criança possibilitam mais dados a respeito de seu desempenho pragmático. A análise do uso do protocolo para acompanhar os resultados de seis meses de intervenção também possibilitou a identificação de correlações relevantes. Apenas uma das 29 questões não apresentou associação com nenhuma das variáveis estudadas. Conclusão: Os resultados obtidos até o momento não são suficientes para determinar que o uso isolado do Protocolo de Avaliação de Habilidades Pragmáticas de Crianças com Transtorno do Espectro do Autismo fornece todos os elementos necessários para a avaliação ou o acompanhamento da intervenção fonoaudiológica.

Palavras-chave: Fonoaudiologia; Comunicação social; Diagnóstico; Transtorno autístico; Linguagem; Criança

\begin{abstract}
Purpose: To propose a protocol to the assessment of pragmatic abilities of children with autism spectrum disorders, test it and compare the results with those of the Functional Communicative Profile. Methods: Participants were 62 children, ages 2 to 12 years without prior diagnosis of any sensorial loss or genetic syndrome. Based on video samples, speech-language pathologists answered to the proposed protocol. The results were analyzed according to their association with other protocols used in the service where the study was carried-out. Results: Only data about communication interactivity and use of verbal communicative mean presented significant correlations with the pragmatic aspects of language performance verified by the proposed protocol. The protocol allowed the supposition that larger experience with the child may provide more information about the child's pragmatic performance. The analysis of the use of the protocol to verify the outcomes of six-month language intervention processes also lead to relevant correlations. Just one of the 29 questions did not result in associations with any of the studied variables. Conclusion: So far, the results are not enough to consider that the isolated use of this tool will provide the necessary information to language assessment of intervention follow-up.
\end{abstract}

Keywords: Speech, language and hearing sciences; Social communication; Diagnosis; Autistic disorder; Language; Child

Trabalho realizado na Faculdade de Medicina, Universidade de São Paulo - USP - São Paulo (SP), Brasil.

${ }^{1}$ Curso de Fonoaudiologia, Faculdade de Medicina, Universidade de São Paulo - USP - São Paulo (SP), Brasil.

Conflito de interesses: Não.

Financiamento: Fundação de Amparo à Pesquisa do Estado de São Paulo - FAPESP - projeto 2016/19811-7.

Autor correspondente: Fernanda Dreux Miranda Fernandes. E-mail: fernandadreux@usp.br

Recebido: Outubro 31, 2020; Aceito: Março 10, 2021 


\section{INTRODUÇÃO}

A avaliação pragmática das habilidades de comunicação, como parte dos procedimentos do diagnóstico fonoaudiológico, desenvolveu-se, no Brasil, a partir da publicação do ABFW Teste de Linguagem Infantil nas áreas de Fonologia, Vocabulário, Fluência e Pragmática ${ }^{(1)}$, que inclui critérios para o delineamento do Perfil Funcional da Comunicação (PFC). Essa publicação resultou no aumento do número de estudos envolvendo os aspectos funcionais da comunicação em diferentes populações, como crianças e adolescentes com síndrome de Down, com deficiência auditiva e com distúrbios específicos de linguagem (DEA), além de estudos com síndromes genéticas.

Sem dúvida, uma área em que o volume de estudos abordando as questões funcionais da comunicação é muito significativo é a que envolve os transtornos do espectro do autismo (TEA). Nessa área, diversos estudos já haviam sido publicados ainda no século passado e continuaram a fornecer evidências científicas para o diagnóstico fonoaudiológico com essa população ${ }^{(2)}$ e para o delineamento e o acompanhamento de processos terapêuticos em linguagem e comunicação.

Por outro lado, o número de estudos envolvendo o desenvolvimento das habilidades pragmáticas em crianças sem alterações de desenvolvimento ou de linguagem é relativamente pequeno $^{(3)}$.

Estudos internacionais recentes continuam a evidenciar a dificuldade para estabelecer modelos uniformes para a avaliação das habilidades pragmáticas. Considerando a noção de que a análise pragmática só faz sentido em amostras de uso da linguagem, a utilização de amostras de comunicação espontânea também é defendida por diversos autores ${ }^{(4,5)}$, que as consideram mais fidedignas para a análise das habilidades individuais, uma vez que conceitos de "apropriado" e "inapropriado" podem variar em diferentes culturas e contextos.

Em contrapartida, a busca por modelos mais objetivos para a avaliação das habilidades pragmáticas destaca-se em estudos que usam diferentes recursos para o registro e a análise dos dados. Outra forma de objetivar a análise pragmática da comunicação é a associação dos aspectos funcionais a determinadas formas ou habilidades linguísticas. Assim, estudos recentes dedicaram-se, por exemplo, a demonstrar que estruturas sintáticas semelhantes podem ser usadas com funções diferentes em diferentes culturas que usam a mesma língua ${ }^{(6)}$; evidenciar que o repertório linguístico interfere nas habilidades pragmáticas ${ }^{(7)}$; verificar o uso de marcadores linguísticos na aquisição de linguagem ${ }^{(8)}$ e estratégias de correção e reparação usadas em diferentes culturas e línguas ${ }^{(9)}$. Indiscutivelmente, porém, os estudos mais enriquecedores em pragmática são exatamente aqueles que levam em conta as diversas interfaces do uso da linguagem ${ }^{(10,11)}$.

Os critérios para o delineamento do Perfil Funcional da Comunicação, publicados no ABFW-Teste de Linguagem Infantil $^{(1)}$ foram determinados a partir da análise da comunicação de crianças com TEA. Considerando-se que as dificuldades com os aspectos pragmáticos da linguagem constituem o elemento central nas dificuldades de comunicação dessas crianças, torna-se natural a busca de aperfeiçoamento desse instrumento também com essa população.

O PFC recomenda a análise de amostras de interação naturalística de, pelo menos, cinco minutos de duração e sua transcrição em um protocolo específico ${ }^{(1)}$. A análise identifica as iniciativas de comunicação, os meios comunicativos utilizados e as funções comunicativas expressas, de um total de 20 possibilidades. Esses procedimentos possibilitam a investigação minuciosa da comunicação de crianças de todas as faixas etárias, o que é útil, não só para o delineamento de um projeto de intervenção individualizado, mas também para o acompanhamento dos resultados dessa intervenção. Entretanto esse é um procedimento trabalhoso, que demanda um tempo de análise relativamente longo, mesmo de profissionais experientes. Após mais de 15 anos de sua publicação inicial, estudos subsequentes indicaram que o PFC oferece elementos clínicos relevantes para a análise e a intervenção nos aspectos pragmáticos da comunicação de crianças $^{(12,13)}$. Entretanto, sua utilização quotidiana na clínica fonoaudiológica parece ter ficado restrita à clínica privada ${ }^{(14)}$ e aos estudos científicos ${ }^{(15,16)}$.

Recentemente foram disponibilizados softwares de transcrição de imagens videogravadas que podem contribuir para estudos multimodais da linguagem ${ }^{(17)}$. Recursos como o Eudico Language Annotador ${ }^{(18)}$ e o Computerized Language Analysis ${ }^{(19)}$ representam, sem dúvida, grandes contribuições para as pesquisas em linguagem, facilitando a sistematização de registros de elementos comunicativos que incluem, além da fala, informações sobre contexto e comunicação não verbal. Entretanto, sua aplicabilidade clínica é bastante reduzida, na medida em que exige a inclusão das informações no sistema, um procedimento tão trabalhoso quanto o proposto pelo PFC.

Assim, um instrumento mais simples, que possa ser utilizado na prática profissional rotineira e que verifique as principais habilidades pragmáticas da comunicação, norteando processos de intervenção e possibilitando seu acompanhamento, representaria uma contribuição relevante para a prática fonoaudiológica baseada em evidências.

Desta forma, os objetivos deste estudo foram propor um protocolo de avaliação das habilidades pragmáticas da comunicação de crianças autistas, aplicá-lo e verificar a associação dos resultados com os obtidos no PFC, na medida em que os dois instrumentos baseiam-se nas mesmas noções a respeito da comunicação de crianças com diagnóstico inserido no espectro do autismo.

\section{MÉTODO}

O estudo foi aprovado pelo Comitê de Ética em Pesquisa da Faculdade de Medicina da Universidade de São Paulo - CEP/ FMUSP, sob número 61204316.1.0000.0065 e os participantes só foram incluídos na pesquisa depois que um de seus responsáveis assinou o Termo de Consentimento Livre e Esclarecido.

\section{Participantes}

Todos os participantes eram atendidos em um serviço de Fonoaudiologia específico para crianças do espectro do autismo, associado ao Curso de Fonoaudiologia da Faculdade de Medicina da Universidade de São Paulo (FMUSP), que é referência nacional na área há mais de três décadas. Foram considerados critérios de inclusão:

- diagnóstico incluído no espectro do autismo, por um neurologista ou psiquiatra, segundo os critérios do DSM-IV ${ }^{(20)}$ ou do DSM-5 ${ }^{(21)}$;

- idade entre 2 e 12 anos.

Foram considerados critérios de exclusão:

- presença de deficiências sensoriais diagnosticadas;

- presença de síndromes genéticas diagnosticadas. 


\section{Material}

O PFC, como proposto pelo $\mathrm{ABFW}^{(1)}$, é usado rotineiramente no Laboratório de Investigação Fonoaudiológica nos Distúrbios do Espectro do Autismo (LIF-DEA) na avaliação inicial e em avaliações semestrais, para o acompanhamento dos processos de intervenção.

O Protocolo de Avaliação de Habilidades Pragmáticas de Crianças com Transtornos do Espectro do Autismo (PAHPEA) foi desenvolvido de forma a incluir informações sobre os mesmos aspectos do desempenho pragmático: iniciativa de comunicação; interatividade da comunicação; meios comunicativos utilizados; diversidade funcional e habilidades discursivas.

O protocolo proposto (Anexos 1 e 2) tem 29 questões que deveriam ser respondidas em uma escala de tipo Likert por um fonoaudiólogo que conhecesse a criança há, pelo menos três meses, e por um colaborador, também fonoaudiólogo, que deveria responder ao mesmo protocolo com base na observação de um segmento de cinco minutos de interação com o fonoaudiólogo, gravado em vídeo.

\section{Procedimento}

O procedimento do estudo envolveu a solicitação de respostas ao Protocolo de Avaliação das Habilidades Pragmáticas de Crianças com Transtornos do Espectro do Autismo (PAHPEA) a colaboradores, também fonoaudiólogos atuantes no mesmo serviço. Colaboraram dez fonoaudiólogos com diferentes níveis e características de formação em pós-graduação, com o objetivo de verificar a confiabilidade do protocolo.

Foram obtidas respostas ao PAHPEA pelo terapeuta e por um fonoaudiólogo colaborador, referentes a todos os participantes, três meses após o início do atendimento fonoaudiológico, com o objetivo de avaliar a consistência das respostas fornecidas por dois examinadores independentes.

Depois de um período de seis meses após a primeira aplicação do PAHPEA, os terapeutas responderam novamente a esse protocolo para todas as crianças que tinham frequentado, pelo menos, $80 \%$ das sessões terapêuticas previstas, com o objetivo de verificar a possibilidade de utilização desse instrumento para o acompanhamento de resultados da intervenção.

Como parte da rotina de acompanhamento do processo de intervenção fonoaudiológica no LIF-DEA, são filmadas amostras de 15 minutos de situações de brincadeira entre a criança e o fonoaudiólogo responsável pelo atendimento. Cada uma dessas amostras foi analisada da seguinte forma:

1 - foram identificados os cinco minutos de interação mais simétrica, que constituíram o corpus desta pesquisa;

2 - esse corpus foi analisado, pelo terapeuta, quanto ao Perfil Funcional da Comunicação, na forma proposta pelo $\mathrm{ABFW}^{(1)}$, também um procedimento rotineiro no LIF-DEA;

3 - o terapeuta e um fonoaudiólogo colaborador aplicaram o Protocolo de Avaliação de Habilidades Pragmáticas de Crianças com Transtornos do Espectro do Autismo - PAHPEA.

4 - seis meses após essas análises, foi realizada nova filmagem da criança em situação de interação lúdica com o fonoaudiólogo terapeuta e os terapeutas responderam novamente ao PFC e ao PAHPEA, em relação a todas as crianças que frequentaram, pelo menos, $80 \%$ das sessões previstas.

\section{Análise dos dados}

Os resultados do PFC e do protocolo de observação foram comparados por meio de análise estatística, utilizando o teste de correlação de Pearson. As principais questões envolveram a associação entre o PFC e o PAHPEA e a identificação dos elementos mais relevantes do PAHPEA para a determinação dessas associações.

\section{RESULTADOS}

Participaram deste estudo 62 crianças com idades entre 2 e 12 anos, atendidas no LIF-DEA da FMUSP.

Os primeiros resultados compararam, em cada questão, as respostas atribuídas a cada participante pelos dois fonoaudiólogos (terapeuta e colaborador). Na Tabela 1, estão apresentados os resultados da análise de correlação de Pearson para as respostas atribuídas pelo terapeuta e pelo colaborador.

As questões 3 e 15 foram as que geraram correlações mais fortes. Elas dizem respeito, respectivamente, ao uso da fala como forma de comunicação e à utilização de estruturas frasais complexas.

Tabela 1. Respostas atribuídas pelo terapeuta e pelo colaborador, segundo a análise de correlação de Pearson

\begin{tabular}{|c|c|c|c|c|c|}
\hline Questão & (valor de p) & Questão & (valor de p) & Questão & (valor de p) \\
\hline 1 & 0,395212 & 11 & 0,337603 & 21 & 0,437627 \\
\hline 2 & 0,523203 & 12 & 0,594548 & 22 & 0,422829 \\
\hline 3 & $0,849688^{\star}$ & 13 & 0,575509 & 23 & 0,520999 \\
\hline 4 & 0,394704 & 14 & 0,484557 & 24 & 0,561006 \\
\hline 5 & 0,593322 & 15 & $0,712813^{*}$ & 25 & 0,62237 \\
\hline 6 & 0,682951 & 16 & 0,360517 & 26 & 0,633468 \\
\hline 7 & 0,555534 & 17 & 0,445447 & 27 & 0,612334 \\
\hline 8 & 0,601625 & 18 & 0,526115 & 28 & 0,510037 \\
\hline 9 & 0,430975 & 19 & 0,326699 & 29 & 0,404926 \\
\hline 10 & 0,611521 & 20 & $0,065135^{\#}$ & & \\
\hline
\end{tabular}

*correlação significativa 
Por outro lado, a questão 20 - que pergunta sobre a habilidade da criança para deixar claro, de forma adequada, quando não quer fazer alguma coisa - não gerou correlação.

A Tabela 2 sintetiza os resultados a respeito da comparação das respostas dos terapeutas em dois momentos do processo de intervenção: 3 meses após o início (para permitir que o terapeuta conhecesse a criança) e após 9 meses do início da intervenção, ou seja, 6 meses após a primeira coleta de dados.

A análise estatística desses dados indicou que, quando o desempenho de cada criança foi analisado em relação à pontuação total nos dois momentos de coleta de dados, foi observada uma diferença significativa em 9\% (p-valor 0,09225). Embora não seja considerada uma diferença estatisticamente significativa, as grandes diferenças individuais verificadas entre crianças com TEA exige a ponderação da relevância desses resultados.

$\mathrm{Na}$ análise individual de cada questão, menos da metade das questões $(1,2,3,5,12,16,19,20,22,23,24,25$ e 27) possibilitou a identificação de diferenças estatisticamente significativas entre os dois momentos de coleta de dados.

A seguir, procedeu-se à verificação da existência de correlação entre o desempenho no primeiro momento de coleta de dados e as eventuais diferenças nas habilidades pragmáticas de linguagem, observadas pelos terapeutas, entre os dois momentos de coleta de dados.

Apenas os dados referentes à proporção de interatividade da comunicação (Tabela 2) e do percentual de uso do meio comunicativo verbal (Tabela 3), como verificados no PFC, apresentaram correlações significativas com o desempenho em linguagem, como observado no PAHPEA.

\section{DISCUSSÃO}

A análise inicial dos dados referentes ao PAHPEA, no presente estudo, revelou que, em apenas três das 29 questões propostas,

Tabela 2. Correlação entre o percentual da interatividade da comunicação verificado no Perfil Funcional da Comunicação e o progresso em seis meses de intervenção verificado no Protocolo de Avaliação de Habilidades Pragmáticas de Crianças com Transtornos do Espectro do Autismo, segundo a análise de correlação de Pearson

\begin{tabular}{ccc}
\hline Questão & Valor de $\mathbf{p}$ & Correlação \\
\hline Q3 & $6,89 \mathrm{E}-05$ & 0,5324 \\
Q7 & $3,00 \mathrm{E}-06$ & 0,6067 \\
Q8 & $1,18 \mathrm{E}-05$ & 0,5766 \\
Q10 & $2,96 \mathrm{E}-07$ & 0,6517 \\
Q13 & $7,11 \mathrm{E}-05$ & 0,5316 \\
Q15 & $2,59 \mathrm{E}-07$ & 0,6541 \\
Q18 & $8,61 \mathrm{E}-05$ & 0,5264 \\
Q23 & $2,12 \mathrm{E}-04$ & 0,5008 \\
Q24 & $1,67 \mathrm{E}-05$ & 0,56824 \\
Q25 & $3,16 \mathrm{E}-06$ & 0,60567 \\
Q27 & $1,50 \mathrm{E}-04$ & $-0,5108$ \\
Q27 & $1,50 \mathrm{E}-04$ & 0,5108 \\
Q28 & $1,39 \mathrm{E}-04$ & 0,513 \\
Total & $3,54 \mathrm{E}-06$ & 0,6032 \\
\hline
\end{tabular}

foram obtidos, ao mesmo tempo, resultados expressivos: semelhança entre as respostas do terapeuta e do colaborador; diferenças significativas entre o primeiro e o segundo momento de coleta de dados; associação com o percentual de interatividade da comunicação e com o uso do meio comunicativo verbal. Essas questões envolvem o uso da fala para comunicação e a habilidade para narrar fatos passados ou histórias e para comentar eventos presentes. As associações entre o uso da comunicação verbal e das habilidades de narrativa com o desempenho e o prognóstico de desenvolvimento de crianças com TEA têm sido sistematicamente descritas na literatura ${ }^{(20)}$. Alguns pesquisadores ${ }^{(19)}$ afirmam que as habilidades de narrativa estão na interface entre o desenvolvimento cognitivo, social e linguístico relacionado ao engajamento social.

Quando consideradas apenas a associação significativa entre o PFC e o progresso no PAHPEA, foi observada correlação relevante em nove questões. Além do uso do meio comunicativo verbal e das habilidades de narrativa, essas questões envolvem respostas a perguntas simples; uso de estruturas complexas para responder; produção de comentários; uso de frases completas e estruturas complexas e troca de turnos na comunicação. Esses mesmos aspectos têm sido investigados por outros pesquisadores, com resultados semelhantes, como um protocolo para a observação de habilidades pragmáticas que aborda as áreas de iniciativa de comunicação, responsividade, comunicação não verbal, atenção socioemocional, funções executivas e negociação(22).

Quando analisadas apenas as associações entre a evolução identificada pelo PAHPEA e o uso do meio comunicativo verbal na primeira avaliação, foram identificadas correlações relevantes em 18 questões. Além dos aspectos mencionados acima, as

Tabela 3. Correlação entre o percentual do uso do meio comunicativo verbal verificado no Perfil Funcional da Comunicação e o progresso em seis meses de intervenção verificado no Protocolo de Avaliação de Habilidades Pragmáticas de Crianças com Transtornos do Espectro do Autismo, segundo a análise de correlação de Pearson

\begin{tabular}{ccc}
\hline Questão & Valor de p & Correlação \\
\hline Q2 & $1,01 \mathrm{E}-05$ & 0,5799 \\
Q3 & $9,48 \mathrm{E}-12$ & 0,7896 \\
Q5 & $6,98 \mathrm{E}-07$ & $-0,6359$ \\
Q6 & $1,01 \mathrm{E}-06$ & 0,6287 \\
Q7 & $1,68 \mathrm{E}-07$ & 0,6616 \\
Q8 & $3,44 \mathrm{E}-07$ & 0,649 \\
Q10 & $7,06 \mathrm{E}-07$ & 0,6356 \\
Q12 & $2,57 \mathrm{E}-07$ & 0,6542 \\
Q13 & $6,20 \mathrm{E}-08$ & 0,6783 \\
Q15 & $1,21 \mathrm{E}-06$ & 0,6253 \\
Q16 & $2,45 \mathrm{E}-05$ & 0,559 \\
Q18 & $2,38 \mathrm{E}-06$ & 0,6116 \\
Q19 & $8,67 \mathrm{E}-05$ & 0,5262 \\
Q20 & $2,06 \mathrm{E}-04$ & 0,5017 \\
Q23 & $1,20 \mathrm{E}-05$ & 0,576 \\
Q24 & $8,58 \mathrm{E}-07$ & 0,6319 \\
Q25 & $1,25 \mathrm{E}-06$ & 0,6246 \\
Q29 & $1,97 \mathrm{E}-05$ & 0,5643 \\
Total & $4,93 \mathrm{E}-09$ & 0,7164 \\
\hline
\end{tabular}


questões abordam a interação; o uso de gestos; a eficiência da comunicação; o uso de pedidos de ação e de informação; jogo simbólico; iniciativa de comunicação e uso de variações em expressões faciais e em prosódia. Por outro lado, duas questões apresentaram correlações significativas apenas entre a proporção da interatividade da comunicação no início da coleta de dados e a evolução identificada no PAHPEA. Foi identificada correlação negativa com o hábito da criança de brincar isolada, em atividades repetitivas e correlação positiva para a atenção e compreensão de variações em expressões faciais e variações de prosódia. Diversos estudos têm associado os resultados da intervenção às habilidades de compreensão da comunicação não verbal, uso de material lúdico e habilidades cognitivas aos resultados da intervenção ${ }^{(23-26)}$.

Diversos pesquisadores têm abordado temas específicos na proposição de formas de avaliar as habilidades de comunicação em crianças com TEA ${ }^{(27)}$, com outros distúrbios do desenvolvimento ${ }^{(28)}$ e em desenvolvimento típico ${ }^{(11)}$. As propostas envolvem a utilização de entrevistas ${ }^{(18)}$, questionários ${ }^{(8)}$ e amostras de comunicação espontânea ${ }^{(29)}$. Diversas variáveis podem interferir na identificação das habilidades e dificuldades com os aspectos pragmáticos da comunicação, como o profissional que faz a análise ${ }^{(30)}$, o contexto de avaliação e de estimulação e as diferentes expressões de uma mesma língua ${ }^{(9)}$. A diversidade de propostas comprova o fato de que ainda não foi estabelecido um procedimento definitivo para a coleta de dados a respeito das habilidades pragmáticas de crianças.

$\mathrm{Na}$ comparação entre as respostas fornecidas pelo terapeuta e pelo colaborador, ficou evidenciado que as questões em que foram observadas diferenças significativas são aquelas em que o contato mais amplo com a criança possibilita a identificação dos comportamentos, como olhar para o adulto, usar sons não verbais na comunicação e expressar prazer, medo ou descontentamento, de forma clara.

A análise das diferenças observadas entre o início do processo de intervenção e os dados obtidos após seis meses de intervenção fonoaudiológica, segundo as observações do terapeuta, identificou diferenças significativas em 13 questões que envolviam: olhar e interagir com o adulto; usar fala e gestos na comunicação; produzir pedidos de informação e de ação; fazer jogo simbólico; expressar-se com clareza; gestos, emissões ou comportamentos descontextualizados e/ou não funcionais; isolamento; habilidades de narrativa e iniciativa e de comunicação. A associação de cada um desses elementos com os resultados da intervenção deve ser considerada individualmente.

Apenas a questão que abordava a inclusão do adulto na brincadeira não apresentou correlação com nenhuma das variáveis estudadas. Entretanto, sugere-se a sua manutenção no protocolo para verificar as respostas envolvendo outros grupos de crianças.

As limitações do presente estudo compreenderam, principalmente, o número restrito de participantes. O número de crianças avaliadas e de fonoaudiólogos aplicando o protocolo seguramente não possibilitou conclusões que autorizem o estabelecimento de parâmetros. Serão necessários novos estudos, em diferentes regiões do país, para que dados mais consolidados sejam obtidos.

\section{CONCLUSÃO}

Embora diversos pesquisadores e clínicos continuem a se dedicar ao desenvolvimento de instrumentos para a avaliação das habilidades pragmáticas de comunicação, ainda não há um método único que associe a especificidade necessária, a facilidade de aplicação e a possibilidade de identificação de variáveis como língua, cultura, interlocutores e contexto.

Os objetivos do presente estudo abrangeram a proposição e a aplicação do PAHPEA e a comparação dos resultados com os do PFC, na medida em que os dois instrumentos baseiam-se nas mesmas noções a respeito da comunicação de crianças com diagnóstico inserido no espectro do autismo.

A proposta do PAHPEA teve por objetivo um protocolo de avaliação das habilidades pragmáticas que fosse simples de usar e que pudesse funcionar como um elemento para o acompanhamento dos resultados da intervenção. Evidentemente, os resultados obtidos até o momento não são suficientes para determinar que o uso isolado desse instrumento fornece todos os elementos necessários para a avaliação ou o acompanhamento da intervenção fonoaudiológica. Ele deve ser usado em conjunto com outros instrumentos já consolidados. Embora uma das questões não tenha apresentado nenhuma associação com nenhuma das variáveis estudadas, sugere-se a sua manutenção no protocolo para verificar as respostas envolvendo outros grupos de crianças.

\section{REFERÊNCIAS}

1. Andrade CR, Befi-Lopes DM, Fernandes FD, Wertzner HF. ABFW: teste de linguagem infantil nas áreas de fonologia, vocabulário, fluência e pragmática. Carapicuiba: Profono; 2000.

2. Varanda CAV, Mendes EVCS, Crescenti MGGM, Nascimento RCGVO, Grillo KRJ, Fernandes FDM. Early identification and intervention on language deficits and behavioral difficulties in early childhood education. Psicol, Teor Pesqui. 2019;35:e35313. http:// dx.doi.org/10.1590/0102.3772e35313.

3. Bretanha AC, Lopes-Herrera AS. Estudo sobre a fidedignidade de dados na avaliação pragmática em crianças com desenvolvimento típico de linguagem. J Soc Bras Fonoaudiol. 2012;24(3):276-81. http://dx.doi. org/10.1590/S2179-64912012000300014. PMid:23128177.

4. Schneider KP. Appropriate behavior across varieties of English Klaus P. J Pragmatics. 2012;44(4):1022-37. http://dx.doi.org/10.1016/j. pragma.2011.09.015.

5. Verbuk A. Developmental evidence against the theoretical distinction between Horn and pragmatic scales. J Pragmatics. 2012;44(4):1680700. http://dx.doi.org/10.1016/j.pragma.2012.07.007.

6. Southwood F. Toward a dialect-neutral assessment instrument for the language skills of afrikaans-speaking children: the role of socioeconomic status. J Child Lang. 2013;40(2):415-37. http://dx.doi. org/10.1017/S0305000912000037. PMid:22357408.

7. Cascia JA, Barr JJ. Association Among Vocabulary, Executive Functions Skills and Empathy in Individuals with autism spectrum disorders. J Appl Res Intellect Disabil. 2017;30(4):627-37. http:// dx.doi.org/10.1111/jar.12257. PMid:27137895.

8. Howard SJ, Melhuish E. An early years toolbox for assessing early executive function, language, self-regulation and social development: validity, reliability and preliminar norms. J Psychoeduc Assess. 
2017;35(3):255-75. http://dx.doi.org/10.1177/0734282916633009. PMid:28503022

9. Filippova E. Developing appreciation of irony in Canadian and Czech discourse. J Pragmatics. 2014;74:209-23. http://dx.doi.org/10.1016/j. pragma.2014.09.003.

10. Ash AC, Redmond SM, Timler GR, Kean J. The influence of scale structure and sex on parental reports of children's social (pragmatic) communication symptoms. Clin Linguist Phon. 2017;31(4):293-312. http://dx.doi.org/10.1080/02699206.2016.1257655. PMid:27936954.

11. Haebig E, Sterling A, Hoover J. Examining the language Phenotype in children with typical development, specific language impairment and fragile X syndrome. J Speech Lang Hear Res. 2016;59(5):1046-58. http://dx.doi.org/10.1044/2016_JSLHR-L-15-0185. PMid:27618388.

12. Sun IYI, Varanda CA, Fernandes FDM. Stimulation of executive functions as part of the language intervention process with children with ASD. Folia Phoniatr Logop. 2017;69(1-2):78-83. http://dx.doi. org/10.1159/000479586. PMid:29248909.

13. Balestro JI, Amato CAH, Sugawara VM, Gibello I, Segeren L, Fernandes FDM. Relations between the perception of communication difficulties, stress levels and behavior of children with autism spectrum disorders. Psychology. 2016;07(12):1391-6. http://dx.doi.org/10.4236/ psych.2016.712139.

14. Sousa CBV, Fernandes FDM. Programa de Intervenção parental com pais de crianças no espectro do autismo. In: Fernandes FDM, editor. Orientações para famílias de crianças no espectro do autismo. São Paulo: Pro-Fono; 2019.

15. Segeren L, Fernandes FDM. Caracterização de um serviço de referência no atendimento fonoaudiológico a indivíduos com Transtorno do Espectro do Autismo. Audiol Commun Res. 2019;24:1-5. http://dx.doi. org/10.1590/2317-6431-2019-2176.

16. Defense-Netrval DA, Fernandes FDM. A oferta da terapia fonoaudiológica em locais de assistência a indivíduos com Transtornos do Espectro do Autista (TEA). CoDAS. 2016;2(4):1-8. http://dx.doi.org/10.1590/23171782/20162015094.

17. Bezerra JTGM, Silva PMS, Cavalcante MCB. Softwares de transcrição como auxílio para as pesquisas em enfoque multimodal no processo de aquisição de linguagem. Texto Livre. 2016;9(1):77-93. http://dx.doi. org/10.17851/1983-3652.9.1.77-93.

18. The Language Archive. ELAN 4.9 [Internet]. The Netherlands; 2015 [citado em 2016 Abr 15]. Disponível em: http://tla.mpi.nl/tools/tla

19. Carnegie Mellon University. CLANWin 1.0 [Internet]. 2015 [citado em 2016 Abr 15]. Disponível em: http://childes.psy.cmu.edu/
20. APA: American Psychiatric Association. Diagnostic and statistical manual of mental disorders. Washington: APA; 1994.

21. APA: American Psychiatric Association. Diagnostic and statistical manual of mental disorders. Washington: APA; 2014

22. Cordier R, Munro N, Wilkes-Gillan S, Speyer R, Pearce WM. Reliability and validity of the Pragmatics Observational Measure (POM): a new observational measure of pragmatic language for children. Res Dev Disabil. 2014;35(7):1588-98. http://dx.doi.org/10.1016/j. ridd.2014.03.050. PMid:24769431.

23. Diez-Itza E, Martínez V, Pérez V, Fernández-Urquiza M. Explicit oral narrative intervention for students with Williams Syndrome. Front Psychol. 2018;8:2337. http://dx.doi.org/10.3389/fpsyg.2017.02337. PMid:29379455.

24. Helland W, Lundervold AJ, Heimann M, Posserud MB. Stable associations between behavioral problems and language impairments across childhood: the importance of pragmatic language problems. Res Dev Disabil. 2014;35(5):943-51. http://dx.doi.org/10.1016/j. ridd.2014.02.016. PMid:24642228.

25. Whyte EM, Nelson KE. Trajectories of pragmatic and nonliteral language development in children with autism spectrum disorders. J Commun Disord. 2015;54:2-14. http://dx.doi.org/10.1016/j.jcomdis.2015.01.001. PMid:25638464.

26. Korrel H, Mueller KL, Silk T, Anderson V, Sciberras E. Research review: language problems in children with attention déficit hyperactivity disorder: a systematic meta-analytic review. J Child Psychol Psychiatry. 2017;58(6):640-54. http://dx.doi.org/10.1111/ jcpp.12688. PMid:28186338.

27. Vitaskova K, Sebkova L. The variable professional perception in assessment of pragmatic language level in autism spectrum disorders and related developmental difficulties. Procedia Soc Behav Sci. 2017;237:1019-25. http://dx.doi.org/10.1016/j.sbspro.2017.02.145.

28. Ghahari S, Hassani H, Purmofrad M. Pragmatic competency and obsessive-compulsive disorder: a comparative assessment with normal controls. J Psycholinguist Res. 2017;46(4):863-75. http://dx.doi. org/10.1007/s10936-016-9467-6. PMid:28013441.

29. Kover ST, Davidson MM, Sindberg HÁ, Weismer SE. Use of the ADOS for assessing spontaneous expressive language in young children with ASD: a comparison of sampling contexts. J Speech Lang Hear Res. 2014;57(6):2221-33. http://dx.doi.org/10.1044/2014_JSLHR-L-13-0330. PMid:25093577.

30. Sasson NJ, Lam KS, Childress D, Parlier M, Daniels JL, Piven J. The broad autism phenotypoe questionnaire: prevalence and diagnostic classification. Autism Res. 2013;6(2):134-43. http://dx.doi.org/10.1002/ aur.1272. PMid:23427091. 
Anexo 1. PROTOCOLO DE AVALIAÇÃO DE HABILIDADES PRAGMÁTICAS DE CRIANÇAS COM TRANSTORNOS DO ESPECTRO DO AUTISMO - PAHPEA

Fernandes, FDM, 2021

Nome da criança:

Data de nascimento: idade da criança: (entre 2 e 12 anos)

Data da filmagem:

Nome da terapeuta:

\section{QUESTIONÁRIO A SER RESPONDIDO PELO TERAPEUTA}

Responder baseado em sua experiência com a criança no ultimo semestre.

Esta criança:

\begin{tabular}{|c|c|c|c|c|c|}
\hline \multirow{2}{*}{1 - Olha para o adulto } & \multirow[t]{2}{*}{ Sempre } & \multirow[t]{2}{*}{ frequentemente } & \multirow[t]{2}{*}{ Às vezes } & \multirow[t]{2}{*}{ Raramente } & \multirow[t]{2}{*}{ Nunca } \\
\hline & & & & & \\
\hline 2 - Interage com o adulto & & & & & \\
\hline 3 - Usa principalmente a fala para se comunicar & & & & & \\
\hline $\begin{array}{l}4 \text { - Usa principalmente sons não verbais para se } \\
\text { comunicar }\end{array}$ & & & & & \\
\hline 5 - Usa principalmente gestos para se comunicar & & & & & \\
\hline 6 - Se faz entender facilmente & & & & & \\
\hline $\begin{array}{l}7 \text { - Responde a perguntas simples (cadê o } \\
\text { carrinho, o que você quer...) }\end{array}$ & & & & & \\
\hline $\begin{array}{l}8 \text { - Responde a perguntas complexas (por que ele } \\
\text { fez isso? o que você fez na escola?...) }\end{array}$ & & & & & \\
\hline $\begin{array}{l}9 \text { - Responde com palavras isoladas ou frases de } \\
\text { duas palavras }\end{array}$ & & & & & \\
\hline $\begin{array}{l}10 \text { - Responde com frases completas com } \\
\text { estruturas complexas }\end{array}$ & & & & & \\
\hline 11 - Interage para pedir ações ou objetos & & & & & \\
\hline 12 - Pede informações & & & & & \\
\hline 13 - Faz comentários adequados & & & & & \\
\hline $\begin{array}{l}14 \text { - Usa palavras isoladas e frases de duas } \\
\text { palavras para se comunicar }\end{array}$ & & & & & \\
\hline $\begin{array}{l}15 \text { - Usa frases completas e estruturas complexas } \\
\text { para se comunicar }\end{array}$ & & & & & \\
\hline 16 - Dá ordens & & & & & \\
\hline $\begin{array}{l}17 \text { - Expressa prazer, medo ou descontentamento } \\
\text { de forma clara }\end{array}$ & & & & & \\
\hline $\begin{array}{l}18 \text { - Troca turnos comunicativos de forma } \\
\text { adequada }\end{array}$ & & & & & \\
\hline 19 - Brinca de faz de conta & & & & & \\
\hline $\begin{array}{l}20 \text { - Deixa claro quando não quer fazer alguma } \\
\text { coisa de forma adequada }\end{array}$ & & & & & \\
\hline $\begin{array}{l}21 \text { - Usa choro, birra ou agressão quando } \\
\text { frustrada ou para interromper alguma atividade }\end{array}$ & & & & & \\
\hline $\begin{array}{l}22 \text { - Produz fala, sons ou gestos } \\
\text { descontextualizados ou não funcionais }\end{array}$ & & & & & \\
\hline 23 - Inicia comunicação & & & & & \\
\hline 24 - Conta historias ou relata fatos & & & & & \\
\hline $\begin{array}{l}25 \text { - Comenta sobre o que está acontecendo ou } \\
\text { pode acontecer (vai cair..., um, dois, mais um...) }\end{array}$ & & & & & \\
\hline 26 - Inclui o adulto na brincadeira & & & & & \\
\hline 27 - Brinca isolada, em atividades repetitivas & & & & & \\
\hline $\begin{array}{l}28 \text { - É atenta e compreende expressões faciais e } \\
\text { prosódia }\end{array}$ & & & & & \\
\hline $\begin{array}{l}29 \text { - Usa expressões faciais e variações } \\
\text { prosódicas para se expressar }\end{array}$ & & & & & \\
\hline
\end{tabular}


Anexo 2. PROTOCOLO DE AVALIAÇÃO DE HABILIDADES PRAGMÁTICAS DE CRIANÇAS COM TRANSTORNOS DO ESPECTRO DO AUTISMO - PAHPEA

Fernandes, FDM, 2021

Nome da criança:

Data de nascimento: idade da criança: (entre 2 e 12 anos)

Data da filmagem:

Nome da terapeuta:

Nome do colaborador:

\section{QUESTIONÁRIO A SER RESPONDIDO POR UM COLABORADOR (NÃO O TERAPEUTA)}

Responder baseado no que é observado na gravação.

Esta criança:

\begin{tabular}{|c|c|c|c|}
\hline \multirow{2}{*}{1 - Olha para o adulto } & Sempre & Ás vezes & Nunca \\
\hline & & & \\
\hline \multicolumn{4}{|l|}{2 - Interage com o adulto } \\
\hline \multicolumn{4}{|l|}{3 - Usa principalmente a fala para se comunicar } \\
\hline \multicolumn{4}{|l|}{4 - Usa principalmente sons não verbais para se comunicar } \\
\hline \multicolumn{4}{|l|}{5 - Usa principalmente gestos para se comunicar } \\
\hline \multicolumn{4}{|l|}{6 - Se faz entender facilmente } \\
\hline \multicolumn{4}{|l|}{$\begin{array}{l}7 \text { - Responde a perguntas simples (cadê o carrinho, o que } \\
\text { você quer...) }\end{array}$} \\
\hline \multicolumn{4}{|l|}{$\begin{array}{l}8 \text { - Responde a perguntas complexas (por que ele fez isso? } \\
\text { o que você fez na escola?...) }\end{array}$} \\
\hline \multicolumn{4}{|l|}{$\begin{array}{l}9 \text { - Responde com palavras isoladas ou frases de duas } \\
\text { palavras }\end{array}$} \\
\hline \multicolumn{4}{|l|}{$\begin{array}{l}10 \text { - Responde com frases completas com estruturas } \\
\text { complexas }\end{array}$} \\
\hline \multicolumn{4}{|l|}{11 - Interage para pedir ações ou objetos } \\
\hline \multicolumn{4}{|l|}{11 - Pede informações } \\
\hline \multicolumn{4}{|l|}{12 - Faz comentários adequados } \\
\hline \multicolumn{4}{|l|}{$\begin{array}{l}13 \text { - Usa palavras isoladas e frases de duas palavras para } \\
\text { se comunicar }\end{array}$} \\
\hline \multicolumn{4}{|l|}{$\begin{array}{l}14 \text { - Usa frases completas e estruturas complexas para se } \\
\text { comunicar }\end{array}$} \\
\hline \multicolumn{4}{|l|}{15 - Dá ordens } \\
\hline \multicolumn{4}{|l|}{$\begin{array}{l}16 \text { - Expressa prazer, medo ou descontentamento de forma } \\
\text { clara }\end{array}$} \\
\hline \multicolumn{4}{|l|}{17 - Troca turnos comunicativos de forma adequada } \\
\hline \multicolumn{4}{|l|}{18 - Brinca de faz de conta } \\
\hline \multicolumn{4}{|l|}{$\begin{array}{l}19 \text { - Deixa claro quando não quer fazer alguma coisa de } \\
\text { forma adequada }\end{array}$} \\
\hline \multicolumn{4}{|l|}{$\begin{array}{l}20 \text { - Usa choro, birra ou agressão quando frustrada ou para } \\
\text { interromper alguma atividade }\end{array}$} \\
\hline \multicolumn{4}{|l|}{$\begin{array}{l}21 \text { - Produz fala, sons ou gestos descontextualizados ou } \\
\text { não funcionais }\end{array}$} \\
\hline \multicolumn{4}{|l|}{22 - Inicia comunicação } \\
\hline \multicolumn{4}{|l|}{23 - Conta historias ou relata fatos } \\
\hline \multicolumn{4}{|l|}{$\begin{array}{l}24 \text { - Comenta sobre o que está acontecendo ou pode } \\
\text { acontecer (vai cair..., um, dois, mais um...) }\end{array}$} \\
\hline \multicolumn{4}{|l|}{25 - Inclui o adulto na brincadeira } \\
\hline \multicolumn{4}{|l|}{26 - Brinca isolada, em atividades repetitivas } \\
\hline \multicolumn{4}{|l|}{27 - É atenta e compreende expressões faciais e prosódia } \\
\hline $\begin{array}{l}28 \text { - Usa expressões faciais e variações prosódicas para se } \\
\text { expressar }\end{array}$ & & & \\
\hline
\end{tabular}

